\title{
Reactive Double Dividing-Wall \\ Distillation Columns: Structure and Performance
}

Relevant Temperature Profiles of Examples I and II

Lijing Zang, Liang Zhang, Kejin Huang*,

Haisheng Chen, Shaofeng Wang, Yang Yuan, Xing Qian

College of Information Science and Technology,

Beijing University of Chemical Technology, Beijing 100029, P. R. China

${ }^{*}$ To whom correspondence should be addressed. Phone: +86 10 64437805. Fax: +86 1064437805.

E-mail: huangkj@mail.buct.edu.cn (K. Huang). 


\section{RELEVANT TEMPERATURE PROFILES OF EXAMPLE I}

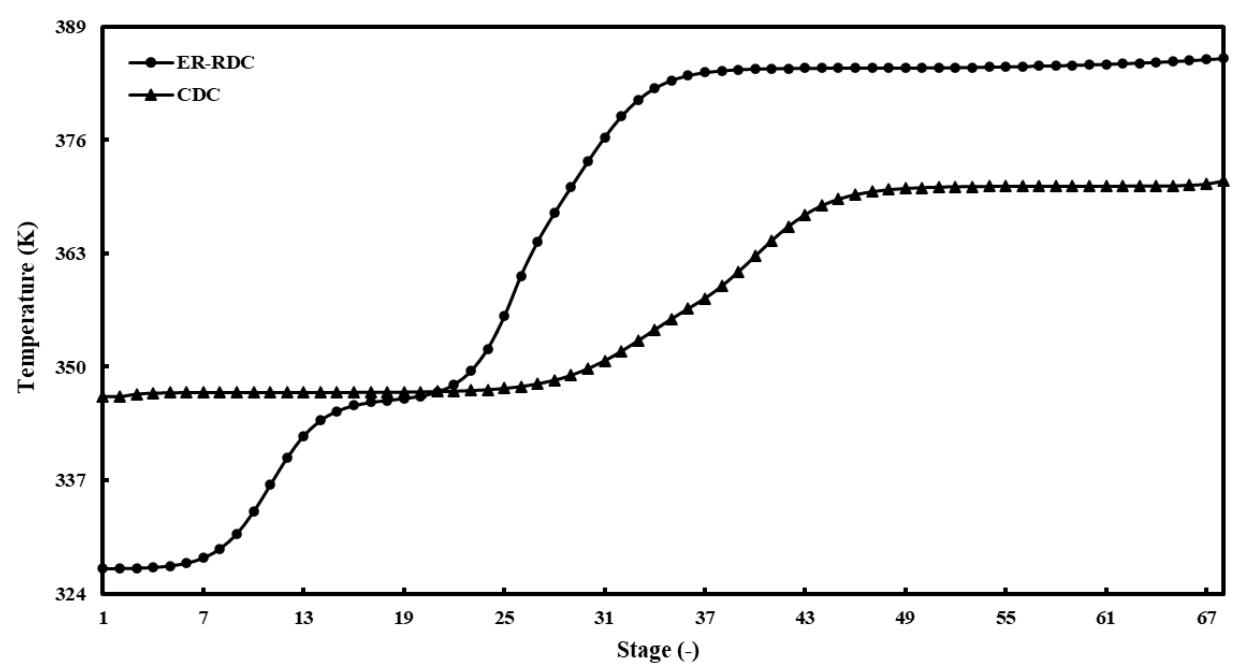

Figure S1. Temperature profile of the ER-RDC+CDC for Example I.

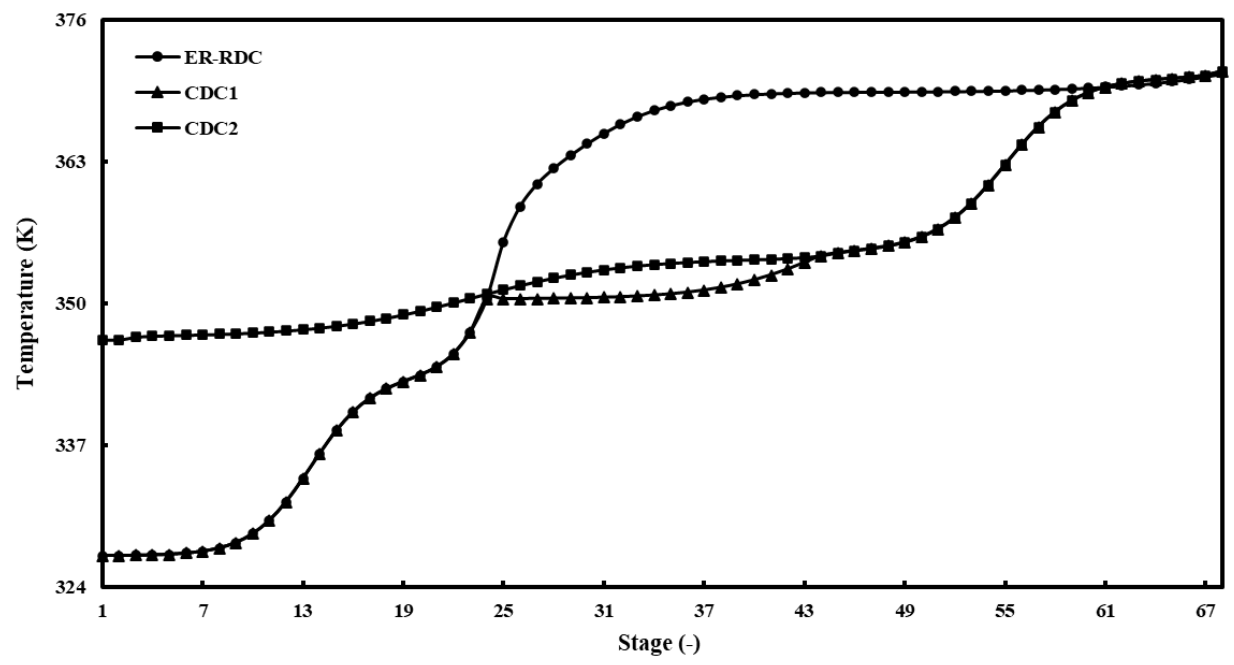

Figure S2. Temperature profile of the R-DDWDC1 for Example I.

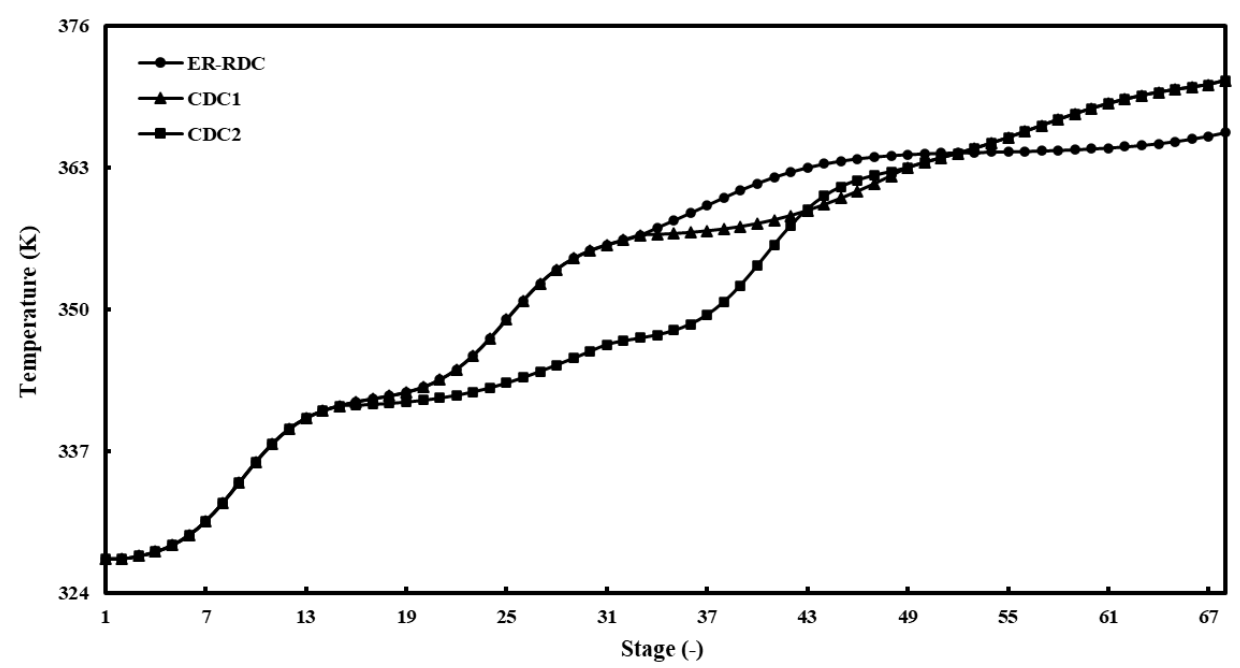

Figure S3. Temperature profile of the R-DDWDC2 for Example I. 


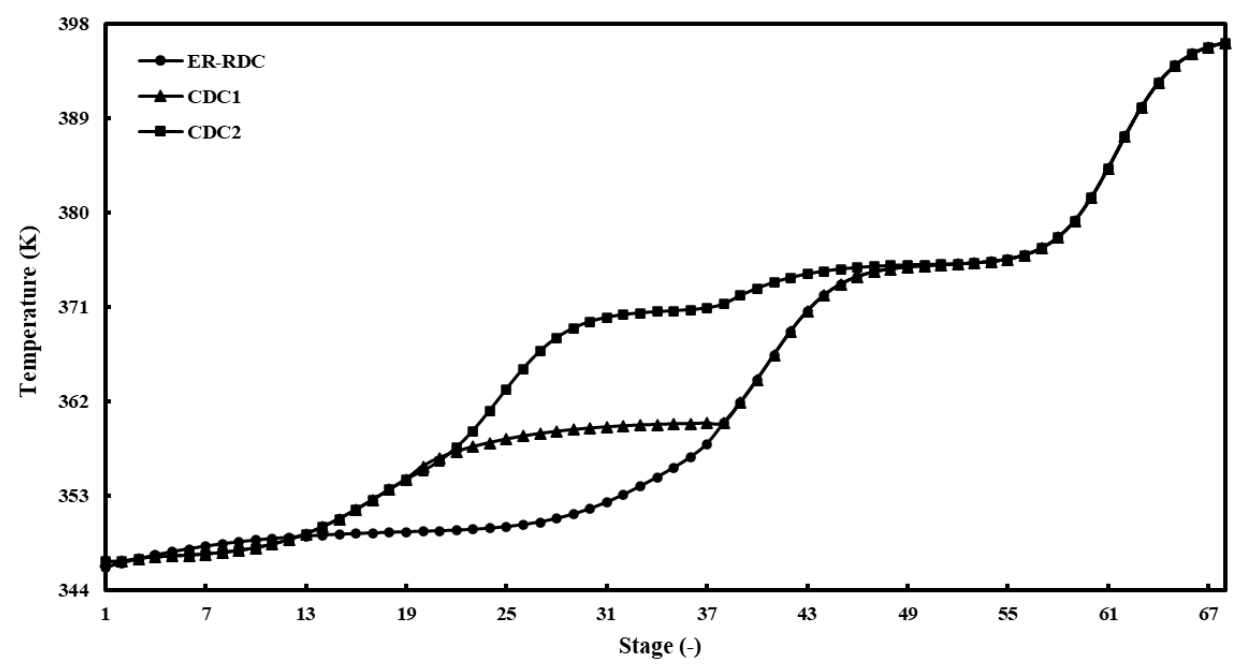

Figure S4. Temperature profile of the R-DDWDC4 for Example I.

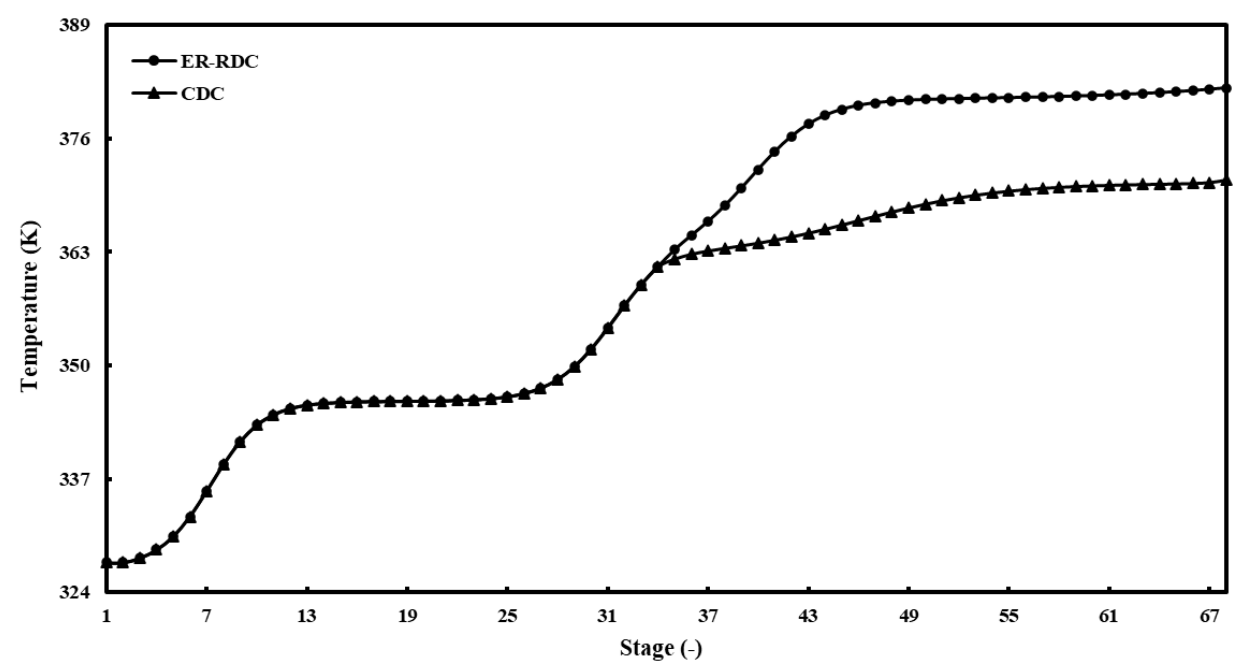

Figure S9. Temperature profile of the R-SDWDC for Example I.

\section{RELEVANT TEMPERATURE PROFILES OF EXAMPLE II}

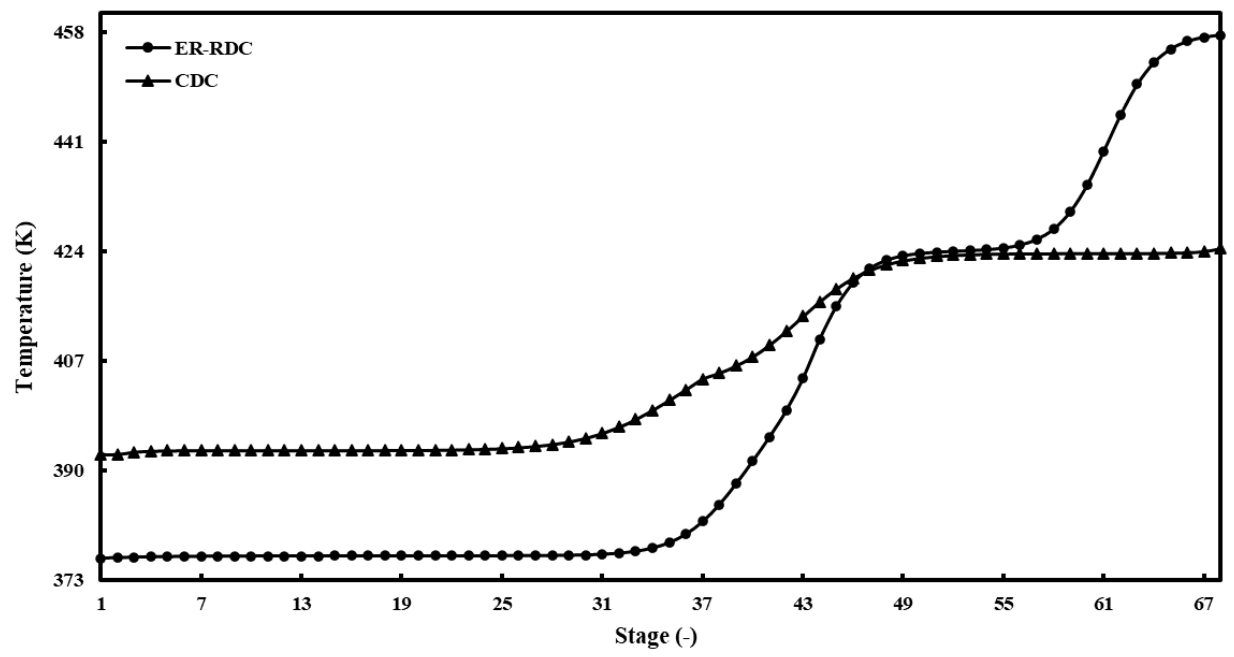

Figure S5. Temperature profile of the ER-RDC+CDC for Example II. 


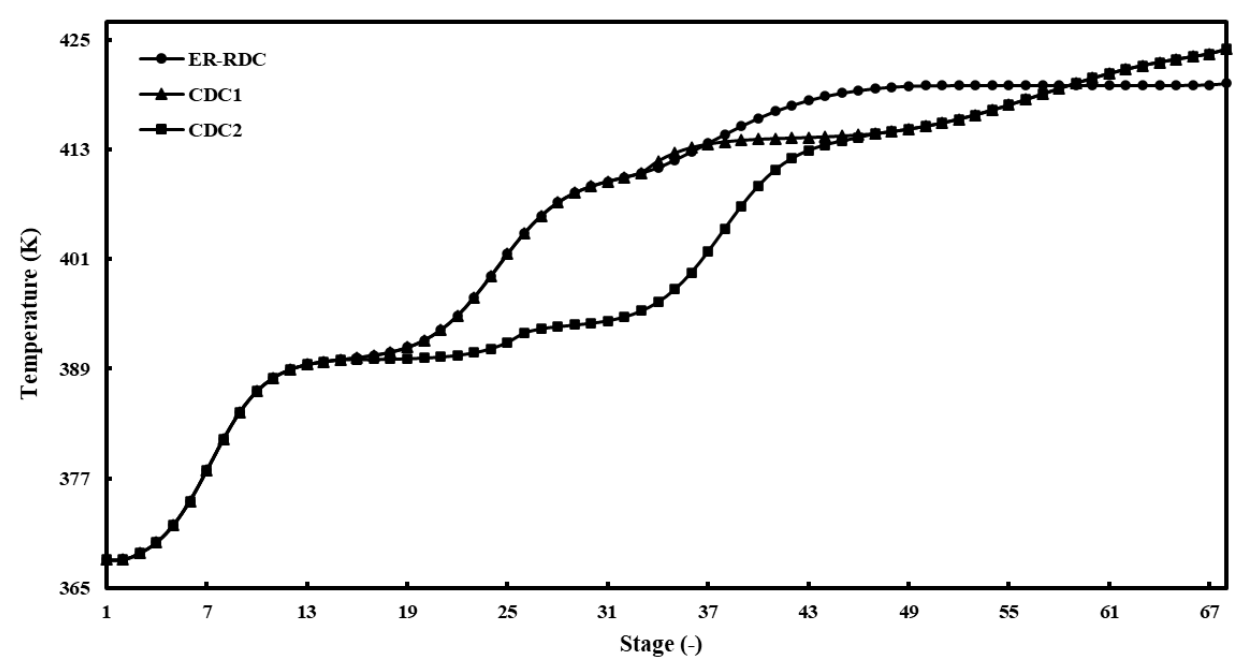

Figure S6. Temperature profile of the R-DDWDC2 for Example II.

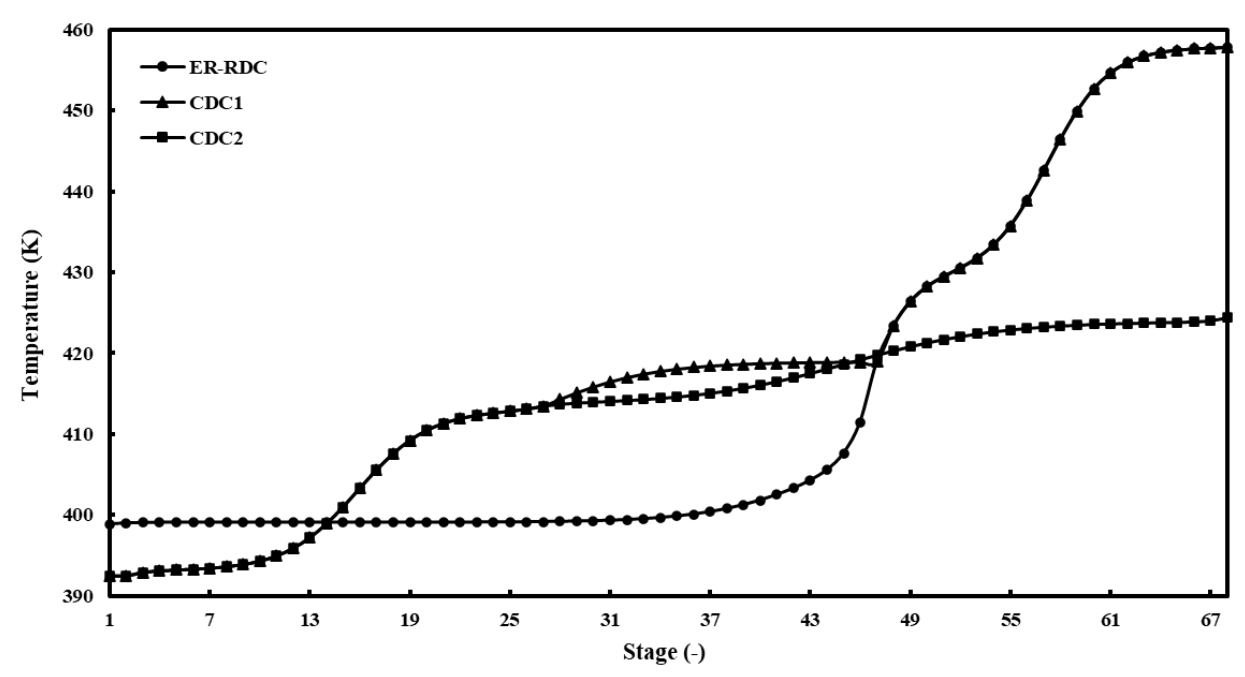

Figure S7. Temperature profile of the R-DDWDC3 for Example II.

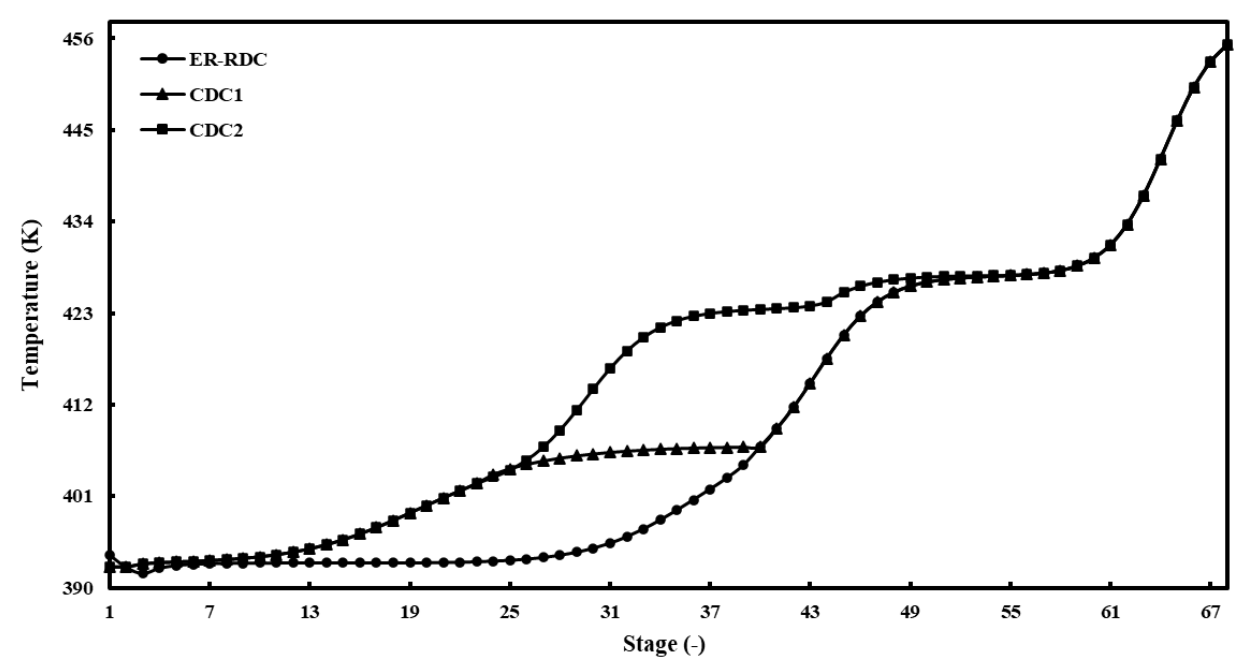

Figure S8. Temperature profile of the R-DDWDC4 for Example II. 


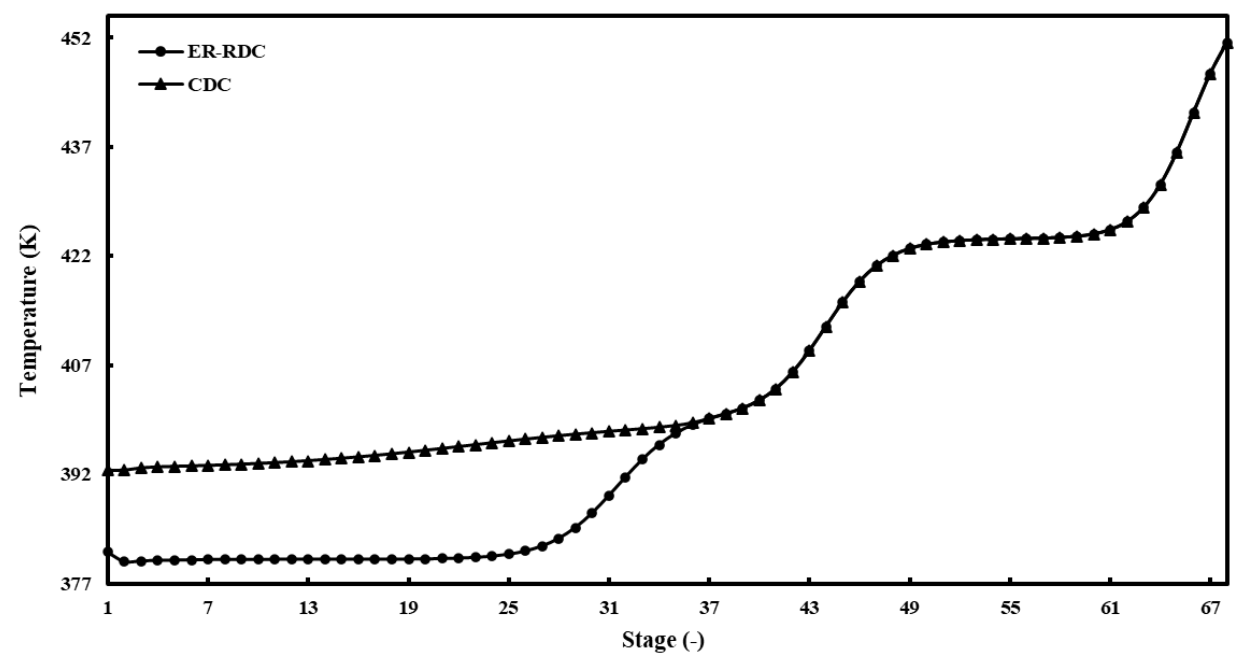

Figure S10. Temperature profile of the R-SDWDC for Example II. 